\title{
Chronic bowel obstruction from colonic stenosis in early infancy-A report of two cases
}

\author{
Sebastian Okwuchukwu Ekenze, Uchechukwu Obiora \\ Ezomike, Ijeoma Esther Nwachukwu, Anthony Ifeanyi \\ Ariom, Kevin Emeka Chukwubuike, Emmanuel Ifeanyi \\ Nwangwu, Uchenna Sunday Onoh, Ebere A Uwah
}

Sub-Department of Paediatric Surgery, College of Medicine, University of Nigeria

Abstract
Bowel obstruction in early infancy may result from a variety of congenital anomalies involving parts of the small and large bowel. However,
in infancy, chronic bowel obstructions from congenital or acquired stenosis of the colon are rare and can cause diagnostic quandary. We
present two cases of an eleven-week old male and a nine-week old male with massive abdominal distension and features of chronic bowel
obstruction dating from neonatal period. In the first case investigations were inconclusive and laparotomy revealed isolated stenosis of
the ascending colon. In the second case colonic stenosis was suspected preoperatively and a barium enema done showed multiple colonic
stenosis confirming our working diagnosis. The diagnostic dilemmas encountered in managing the first patient are discussed to highlight
the need for high index of suspicion of this condition in infants with chronic constipation. The way experience in managing the first case
influenced diagnosis of the second case is also highlighted.

\section{Introduction}

Congenital colonic stenosis is a variant of colonic atresia with varying severity of colonic lumen narrowing ${ }^{1}$. It is a rare congenital anomaly of the gastrointestinal tract with a reported incidence of 1 in 40,000 births $s^{2}$. The aetiology is largely unknown, but the development of the condition has been associated with fetal mesenteric vascular accidents from fetal intestinal kinks, internal herniation, intussusception, volvulus, and maternal use of cocain $e^{2-4}$. Colonic stenosis has also been reported from acquired conditions like necrotizing enterocolitis (NEC), however such stenosis commonly involves multiple areas of the large bowel ${ }^{5,6}$. Reports indicate that any of the segments of the colon may be affected by the congenital stenosis, but it is commoner in the ascending and descending colon ${ }^{1,2,4}$. Congenital colonic stenosis may present early or late in life depending on the severity of the stenosis ${ }^{2,7}$. Reported symptoms include passage of small quantity of faeces, abdominal distension, colicky abdominal pains, occasional vomiting, and failure to gain weight ${ }^{1,2,4,5}$. As a result of this there may be diagnostic uncertainty as most of the reported cases were diagnosed intraoperatively following laparotomy for intestinal obstruction ${ }^{7}$.

We report a case of congenital solitary stenosis involving the ascending colon that presented with severe abdominal distension and intermittent constipation that dated back to the newborn period in an 11-week old male and a second case of multiple long segment strictures involving cecum, ascending colon, transverse colon and descending colon in a 9-week old male. The focus of this article is to highlight the diagnostic challenges encountered in managing these patients, as well as the need to consider the diagnosis of colonic stenosis in infants with intermittent constipation and abdominal distension that commenced during newborn period.

\section{Case Report 1}

An eleven-week old male presented with progressive generalized abdominal distension that commenced during the neonatal period. He was the first of a set of monozygotic twins delivered via emergency Caesarean section at 35 weeks gestational age due to antepartum haemorrhage. There was no history of maternal smoking, cocaine use or ingestion of any other vasoconstrictors. His birth weight was $2.3 \mathrm{~kg}$ and he spontaneously passed meconium within the first 24 hours of life. Subsequently, however, his stools became of small volume with associated abdominal distension and intermittent episodes of non-projectile, non-bilious vomiting. Despite regular feeds, his weight gain lagged behind that of his twin.

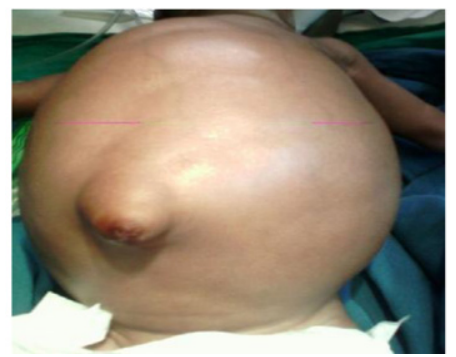

Figure 1: Supine view, plain abdominal radiograph showing markedly dilated bowel loops 


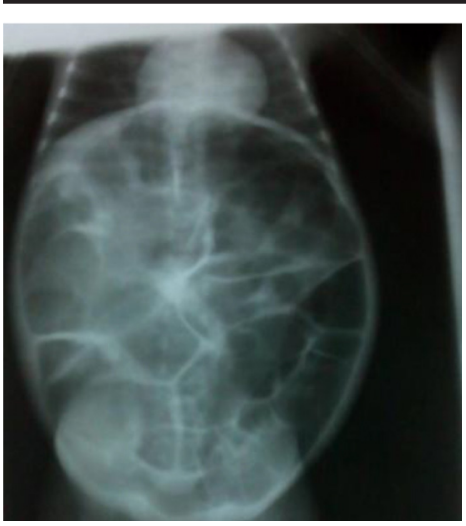

Figure 2: Intra-operative picture showing stenosed ascending colon (left arrowe), dilated caecum and distal ileum(right arrow)

Clinical examination at presentation revealed moderate pallor, weight of $3.2 \mathrm{Kg}$, markedly distended and tense abdomen with visible peristalsis, tympanitic percussion notes and hyperactive bowel sounds. Digital rectal examination was unremarkable.

Laboratory investigations revealed haemoglobin level of 8.6g/dl and normal serum electrolytes. Plain abdominal radiograph showed markedly dilated bowel loops filling the entire abdomen with gas in the rectum (Figure 1). Abdominal sonogram revealed gaseous bowel dilatation with minimal free intraperitoneal fluid. Contrast enema was not done because of the markedly distended and tense abdomen.

An initial attempt was made to decompress the bowel nonoperatively by insertion of dulcolax suppository while patient was placed on appropriate intravenous fluid and nil per oral.. When this failed to increase the stool volume or reduce the abdominal distension, the patient underwent exploratory laparotomy.

Intraoperative findings included stenosis involving $5 \mathrm{~cm}$ of the ascending colon commencing at a point $6 \mathrm{~cm}$ from the ileocaecal valve with marked gaseous dilation of the distal jejunum, ileum and caecum (Figure 2). The proximal jejunum was collapsed and the duodenojejunal junction was at the normal position. A right hemicolectomy with ileotransverse anastomosis was done. Immediate postoperative period was uneventful and patient had good recovery. He has been gaining adequate weight on follow-up.

\section{Case report 2}

A 9-week old male infant who presented with 7 weeks history of recurrent generalized abdominal distension but has been passing normal volume stools daily . There were also histories of failure to thrive, bilateral leg swellings and blood transfusion due probably to nutritional anaemia.

Delivery was via spontaneous vertex delivery at gestational age of about 34 weeks due to preterm onset of labour. There is no history maternal use of cocaine, tobacco, unorthodox medications during pregnancy. His birth weight was $2 \mathrm{~kg}$, he cried immediately after birth, spontaneously passed meconium within the first 24 hours of birth and was fed with breast milk and artificial milk. He was not admitted in newborn special care baby unit. Clinical examination revealed an irritable $2.3 \mathrm{~kg}$ male infant, moderately pale, with grade 5 bilateral pitting lower limb oedema and depressed

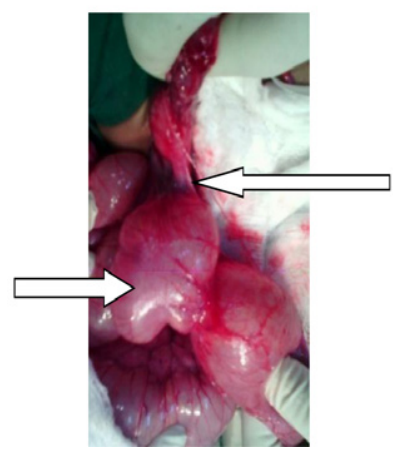

Figure 3: Barium enema showing multiple colonic stenosis in case report 2

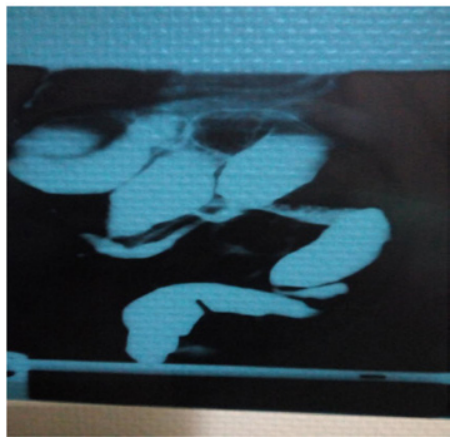

Figure 4: The segment of resected stenosed ascending,transverse and descending colon

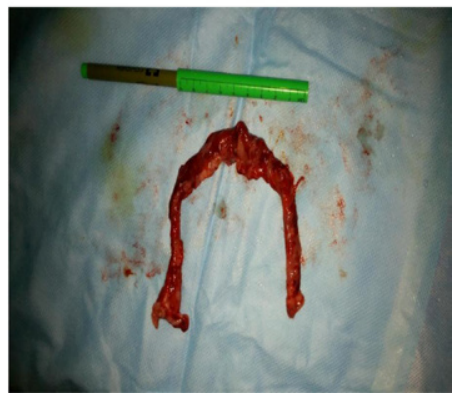

Figure 5: The segment of resected stenosed ascending,transverse and descending colon

Abdominal examination revealed uniformly distended abdomen with visible peristalsis, everted umbilicus, tympanitic percussion notes and hyperactive bowel sounds. Digital rectal examination was unremarkable. Plain abdominal radiograph showed gaseous distension of bowel loops in the entire abdomen and gas in the rectum. Barium enema revealed multiple areas of colonic stenosis [figure 3 ]. Serum electrolytes were within normal limits and haemoglobin estimation was $5.7 \mathrm{~g} / \mathrm{dl}$. He was transfused and optimized prior to surgery.

Patient underwent exploratory laparotomy and intraoperative findings include extensive stenosis involving the caecum, ascending colon, proximal and distal parts of transverse colon and descending colon [figure 4 ]. Patient had resection of stenotic colonic segments with ileo-sigmoid 
colon anastomosis. Postoperatively, patient had uneventful recovery and on follow-up has been gaining adequate weight. Histopathology report on the resected bowel segment excluded Hischsprung's disease.

\section{Discussion}

Colonic stenosis is a rare but established cause of pediatric intestinal obstruction and the presentation may be in neonatal age, early infancy (as in our two case reports) or less commonly in older children ${ }^{2,7}$. They may be congenital or acquired. The congenital types are thought to occur when there is incomplete bowel injury as may be the case when injury occurs close to the bowel wall, allowing collateral blood flow to preserve the injured tissue or when there is limited ischemia, in which the blood supply is partially or intermittently occluded. The acquired types are mainly from strictures following Necrotizing Enterocolitis(NEC) ${ }^{8,9}$.

The factors that determine the time of presentation are largely unknown, but may be related to the severity of the stenosis and whether it is congenital or acquired. The common clinical features in the reported cases include passage of small quantity of faeces, abdominal distension, colicky abdominal pain, occasional vomiting, and failure to gain adequate weight $t^{1,2,4,5,7}$. Although most of these symptoms were exhibited in the index cases, they are not pathognomonic and can be manifestations of other diseases like Hirschsprung's disease, Necrotizing EnteroColitis(NEC), distal small bowel atresia, and intestinal malrotation that may present in the same age group ${ }^{1,5}$. Perhaps it is for this reason that the clinical diagnosis of this disease is difficult, and most of the reported cases were initially managed with the preceding diagnoses pending imaging or laparotomy ${ }^{7}$. Studies have shown that preoperative diagnosis of congenital colonic stenosis may be facilitated by the use of contrast enema and colonoscopy ${ }^{1,2,4,5,10}$. Contrast enema delineates small caliber distal colon and a distended proximal colon in cases of colonic stenosis. It also differentiates colonic stenosis from colonic atresia in which there is a cut- off point between a distal colon filled with contrast and a dilated proximal bowel filled with luminal air.

In the first case we did not obtain a barium enema as there was marked abdominal distension prompting fears that retention of rectally delivered contrast may worsen symptoms or may lead to perforation ${ }^{4}$. In the second case report barium enema revealed multiple colonic stenosis (figure 4). Colonoscopy will reveal an abrupt change in caliber in the affected area of the colon. Definitive treatment of congenital or acquired colonic stenosis is surgery ${ }^{1-5}$. The stenosis may be single( as in the first case report) or multiple(as in second case report $)^{1,4,7}$. It may affect ascending colon, transverse colon, descending colon or sigmoid colon in isolation or in various combinations as multiple stenoses ${ }^{1,2,4}$. It is therefore imperative to thoroughly examine the entire length of the bowel to assess patency and ensure that no affected part of the bowel is missed. Surgical treatment can be undertaken either by open or by laparoscopic procedure ${ }^{1-5,10,11}$ and involves resection of the stenotic segment(s) and primary anastomosis for the right colonic stenosis ${ }^{2}, 10$. This was performed in our first case report. Intraoperatively we resected the stenotic segment as well as adjoining bowel thought to have had precarious blood supply due to the preceding causative vascular accident. These areas with uncertain blood supply may have some degree of hypomotility and if not excised may lead to delayed return of bowel function postoperatively. In left sided colonic stenosis, it may be expedient to perform proximal protective enterostomy after the resection and anastomosis ${ }^{1,5,7}$. In multiple colonic stenosis, enteroplasties may be considered in some cases to avoid the risk of excessive colonic loss with multiple resection ${ }^{1,7}$. In the second case we carried out an extensive large bowel resection involving both right and left colon and with no proximal protective enterostomy. There was also no evidence of short bowel syndrome which may be due to the great ability of neonatal ileum for adaptation. Advances in resuscitation and peri-operative care have led to high survival rates and postoperative period is often uneventful with good outcome in most of the reported cases 12,13 .

\section{Conclusion}

Colonic stenosis is a rare cause of lower intestinal obstruction in early childhood. The clinical manifestations of the disease are not pathognomonic and may mimic some other disorders in this age group. It is imperative to have high index of suspicion for this disease and institute appropriate diagnostic workup in neonates and infants with progressive abdominal distension that dates back to newborn period despite regular passage of small quantity of stools and absence of bilious vomiting.

\section{References}

1. Elisa Z, Cinzia C, Sergio S, Giuseppe LV, Fortunato S. Multiple Congenital Colonic Stenosis:ARare Gastrointestinal Malformation. Case Rep Pediatr 2016; 2016;2016:6329793. doi: 10.1155/2016/6329793.

2. Galván-Montaño A, Suárez-Roa Mde L, Carmona-Moreno E. Congenital stenosis of the colon with foreign bodies. Case report. Cir Cir 2010; 78(3): 259-61.

3. Hajivassiliou CA. Intestinal obstruction in neonatal/pediatric surgery. Semin Pediatr Surg 2003; 12(4): 241 - 53.

4. Hamid R, Shera AH, Bhat NA, Baba A, Rashid A. Congenital Descending Colonic Stenosis with Perforation of Transverse Colon in a Neonate: A Case Report. J. Nepal Paediatr. Soc 2012; 32(1): 73 - 75.

5. Mirza B, Iqbal S, Ijaz L. Colonic atresia and stenosis: our experience. J Neonat Surg 2012; (1):4.

6.Schimpl G,Höllwarth ME,Fotter R, Becker H. Late intestinal strictures following successful

treatment of necrotizing enterocolitis. Acta Paediatr Suppl.1994; 396:80-3.

7. Hamid R, Ali I, Bhat N, Baba AA, Mufti G, Wani SA. Management Congenital Colonic Stenosis. J Neonatal Biol 2015; 4: 166.

8. Heida FH, Loos MH, Stolwijk L, Te Kiefte BJ, van den Ende SJ, Onland $\mathrm{W}$ et al. Risk factors associated with postnecrotizing enterocolitis strictures in infants. J Pediatr Surg. 2016 ;51(7):1126-30. doi: 10.1016/j.jpedsurg.2015.09.015.

9. Burnand KM, Zaparackaite I, Lahiri RP, Parsons G, Farrugia MK, Clarke SA et al. The value of contrast studies in the evaluation of bowel strictures after necrotising enterocolitis. Pediatr Surg Int. 2016 ;32(5):465-70. doi: 10.1007/s00383-016-3880-7.

10.Ruggeri G, Libri M, Gargano T, Pavia S, Pasini L, Tani G, et al. Congenital colonic stenosis: a case of late-onset. Pediatr Med Chir 2009; 31(3): $130-3$.

11. García Vázquez A, Cano Novillo I, Portela Casalod E, Benavent 
Gordo MI, Berchi García FJ. Congenital colonic stenosis. An Esp Pediatr 2002; 56(3): 258 - 60.

12. Phad N, Trivedi A, Todd D, Lakkundi A. Intestinal strictures postnecrotising enterocolitis: clinical profile and risk factors. J Neonatal
Surg. 2014; 20;3(4):44.13.Abu-Judeh HH, Methratta S, Ybasco A,

Garrow E, Ali S. Congenital colonic stenosis. South Med J. 2001; 94(3): 344-6 